\title{
Le presbytère québécois au 19e siècle Espace de vie, lieu de tensions et enjeu de pouvoir
}

\section{Christine Hudon}

\section{Q OpenEdition}

12 Journals

\section{Édition électronique}

URL : http://journals.openedition.org/abpo/1345

DOI : $10.4000 /$ abpo.1345

ISBN : 978-2-7535-1492-8

ISSN : 2108-6443

\section{Éditeur}

Presses universitaires de Rennes

Édition imprimée

Date de publication : 20 décembre 2003

Pagination : 125-138

ISBN : 978-2-86847-933-4

ISSN : 0399-0826

\section{Référence électronique}

Christine Hudon, «Le presbytère québécois au 19e siècle Espace de vie, lieu de tensions et enjeu de pouvoir », Annales de Bretagne et des Pays de l'Ouest [En ligne], 110-4 | 2003, mis en ligne le 20 décembre 2005, consulté le 01 mai 2019. URL : http://journals.openedition.org/abpo/1345 ; DOI 10.4000/abpo. 1345 


\title{
Le presbytère québécois au $19^{\mathrm{e}}$ siècle \\ Espace de vie, lieu de tensions et enjeu de pouvoir ${ }^{1}$
}

\author{
Christine Hudon \\ Professeur d'Histoire contemporaine \\ Université de Sherbrooke
}

On a passablement écrit sur le presbytère. Des études ont démontré l'intérêt architectural de ces maisons souvent à l'avant-garde des styles de l'époque, figurant assurément parmi les demeures les plus belles et les mieux décorées du village ${ }^{2}$. La vie au quotidien à l'intérieur du presbytère demeure toutefois méconnue. On sait peu de chose des conditions de travail des ménagères et de la situation des proches du curé qui habitent parfois avec lui et avec le vicaire, main-d'œuvre cléricale dont le nombre tend à augmenter au cours du $19^{\mathrm{e}}$ siècle. Pourtant, tenter de percer cet espace permet de lever le voile sur les rapports sociaux et les rapports de genre qui prennent place à la fois dans l'Église et dans le monde rural. C'est du moins ce que postule cet article qui vise à révéler, à travers l'analyse des règlements et de la correspondance cléricale évoquant la vie au presbytère, les relations qu'entretiennent entre eux les occupants de cette maison singulière afin d'éclairer trois types de rapports inégalitaires : ceux, qui prennent place, d'abord, parmi le clergé, entre curé et vicaire, ceux perceptibles, à un second niveau, dans l'Église, entre clercs et laïcs, et enfin, ceux qui, au sein de la société du $19^{\mathrm{e}}$ siècle, unissent les hommes et les femmes. Le presbytère est donc ici considéré comme un microcosme de l'univers paroissial, des écarts de condition qui le caractérisent, des représentations et des préjugés qui marquent les différents états de vie et des tensions que ces situations inégales engendrent.

1. La recherche pour cet article a bénéficié du soutien financier du Fonds pour la formation des chercheurs et l'aide à la recherche et du Conseil de recherches en sciences humaines du Canada.

2. SimARD, Jean, Les Arts sacrés au Québec, Boucherville, Éditions de Mortagne, 1989, p. 199-203; MARTIN, Paul-Louis, "Le paysage des noyaux religieux ", COURVILLE, Serge et SÉGuIn, Normand (dir.), Atlas historique du Québec. La paroisse, Sainte-Foy, Presses de l'Université Laval, 2001, p. 75-77. 
À plus d'un égard, le presbytère représente un espace fascinant à étudier. C'est, d'une part, un espace public où viennent les paroissiens ou des visiteurs étrangers en quête de conseils, d'aide matérielle ou de soutien spirituel. Avec sa "salle des habitants ", c'est aussi un lieu communautaire, un point de rencontre où hommes et femmes discutent et se divertissent, les unes à bonne distance des autres et souvent dans des pièces séparées. C'est, d'autre part, une maison privée, où le curé prie, lit son bréviaire, prépare ses instructions et se repose, à l'écart des bruits de la rue. Soucieux de bien circonscrire son espace de vie et son intimité, le clergé de la seconde moitié du $19^{\mathrm{e}}$ siècle tentera d'ailleurs d'écarter les salles communautaires des presbytères pour les établir dans des constructions distinctes, afin de n'être plus inquiété par les rires et les conversations des paroissiens réunis ${ }^{3}$.

Le presbytère sert de logis à plusieurs personnes. On y retrouve, bien sûr, le curé, qui partage généralement sa maison avec une servante et/ou, dans des cas beaucoup plus rares, un serviteur. Plusieurs curés âgés, malades ou titulaires d'une paroisse populeuse bénéficient aussi des services d'un vicaire qui réside, lui aussi, au presbytère. Ce vicaire rétribué par le curé est habituellement un prêtre ordonné depuis quelques mois, tout au plus depuis deux ou trois ans, mais il arrive également qu'un curé déchu, aux prises avec des problèmes d'alcool ou trouvé coupable d'inconduite sexuelle, soit placé chez un confrère de confiance pour expier sa faute et faire de nouveau ses preuves. Des curés hébergent en outre, pour des périodes plus ou moins longues, un ou plusieurs proches, par exemple leurs père et mère, une sœur ou un frère célibataire ou quelque autre parent plus éloigné. D'autres, enfin, donnent le gîte à un protégé. Il y a donc, dans certains presbytères, un ou plusieurs enfants. Tout ce monde de conditions, d'état, de sexe et d'âge différents forme des maisonnées à la taille variable : deux, trois, quatre, voire cinq ou six personnes logent dans la maison curiale.

\section{Le jeu subtil des murmures}

La présence d'un ou plusieurs prêtres et de laïcs dans un même espace pose régulièrement problème aux autorités cléricales. C'est que la population surveille attentivement le presbytère, véritable maison de verre, sur laquelle circulent facilement les commérages. La vie des occupants du presbytère attire en effet l'attention. Le curé est exposé au jugement du public qui pèse et soupèse ses enseignements et sait, au besoin, identifier et exprimer les contradictions entre ses gestes et ses paroles. En cette matière, le curé, gardien de la moralité, celui qui définit, en appliquant le discours de l'Église, l'acceptable et l'inacceptable, celui qui scrute les consciences et stigmatise les inconduites, parfois en les dénonçant du haut de la chaire ${ }^{4}$,

3. Hudon, Christine, Prêtres et fidèles dans le diocèse de Saint-Hyacinthe, Sillery, Septentrion, 1996, p. 99-100.

4. GAGNON, Serge et HARDY, René (dir.), L’Église et le village au Québec : 1850-1930 : l'enseignement des Cahiers de prônes, Montréal, Leméac, 1979. 
celui qui exclut les fautifs de la communion, du cimetière ou même de la communauté s'il les dénonce à l'appareil judiciaire ${ }^{5}$, fait l'objet de médisances et de critiques. La contestation du prêtre, qui s'énonce sur la base des critères moraux dont il se fait lui-même le défenseur, peut ultimement conduire à son retrait de la paroisse ${ }^{6}$. Les secrets de celui qui connaît les secrets de tout un chacun ${ }^{7}$, et, par amalgame, les secrets de ses proches, circulent dans la paroisse et hors de cet espace. En effet, le reste de la maisonnée presbytérale n'est guère plus à l'abri des regards soupçonneux et volontiers inquisiteurs des paroissiens que le curé lui-même. Sous la plume ou dans la bouche de certains accusateurs, la maison presbytérale devient maison de jeux, maison close, maison de débauche. Les rumeurs et les dénonciations qui révèlent au grand jour le quotidien de cette demeure particulière, qui rendent publiques les faits intimes, s'exposent comme une forme de contrôle du détenteur du pouvoir religieux.

Les évêques doivent composer avec cette cohabitation de clercs et de laïcs. Pendant tout le $19^{\mathrm{e}}$ siècle, ils restent à l'affût des murmures pour parer d'éventuels scandales. Les autorités diocésaines tolèrent généralement que les curés hébergent un ou plusieurs proches sans toutefois encourager la chose. Au moindre soupçon sur les mœurs de tel ou tel parent logeant au presbytère, au moindre commérage ou à la moindre plainte à leur sujet, les évêques interviennent. La peur que des comportements délictuels n'entachent la réputation du clergé les rend généralement prompts à écrire au prêtre concerné, à l'abreuver de conseils, à lui adresser des semonces. Tel frère alcoolique, tel autre accusé de vol, telle sœur à la langue trop bien pendue, telle nièce devenue enceinte hors mariage sont priés de quitter le presbytère. Les frasques de la famille du curé rejaillissent immanquablement sur lui. Aussi mineurs soient-ils, les délits des membres de la maisonnée ne sont pas facilement absous par les populations, promptes à accuser de favoritisme et d'incurie les curés qui tolèrent, même de mauvaise grâce, les inconduites de leurs proches.

Parfois, comme dans l'exemple suivant, c'est le comportement du curé, et non celui de ses proches, qui suscite les bavardages des paroissiens. On accuse le prêtre d'être trop lié à certains laïcs qui partagent sa maison, de négliger son ministère, d'afficher une conduite suspecte, peu en accord avec l'engagement sacerdotal. L'évêque Joseph LaRocque apostrophe un curé en ces termes :

5. Sur les rapports entre les curés et les officiers de justice, lire HARDY, René, Contrôle social et mutation de la culture religieuse au Québec, 1830-1930, Montréal, Boréal, 1999, p. $173-186$

6. HuDON, Christine, « Beaucoup de bruits pour rien? Rumeurs, plaintes et scandales autour du clergé dans les paroisses gaspésiennes, 1766-1900 ", Revue d'histoire de l'Amérique française, 55, 2 (automne 2001), p. 217-240.

7. FABRE, Daniel, "Familles. Le privé contre la coutume ", ARIÈs, Philippe et al., De la Renaissance aux Lumières, Histoire de la vie privée, t. 3, Philippe ARIĖs et Georges DuBY (dir.), Paris, Seuil, 1985, p. 578. 
"Vous êtes en proie à des critiques pénibles pour la Religion, pour moi et pour le Clergé. Vous donnez à boire des boissons fortes [...]. Vous vous amusez avec des garçons de votre voisinage [...]. Vous êtes attaché à l'enfant de votre domestique. On vous a vu l'aller chercher dans vos bras, à dix heures du soir, en traversant le village. Vous le faites coucher avec vous ${ }^{8}$."

À cette crainte du scandale qui obsède l'autorité diocésaine s'en ajoute une autre, celle que le presbytère devienne lieu de désordre et de dispute, que les membres de la maisonnée, clercs et laïcs, y soit dissipés ou qu'ils s'y querellent publiquement, que soient brisées, en somme, l'idée d'harmonie associée au corps clérical et l'image de paix et de quiétude que devait idéalement évoquer la maison curiale ${ }^{9}$. Voici l'abbé Pelletier, admonesté par son évêque :

"Faites donc en sorte de vider votre maison de toute la parenté, grands et petits. Laissez-moi vous le dire, votre maison a plutôt l'air d'une habitation de famille que d'un presbytère, et cela me fait peine. [...] Quel ennui pour votre vicaire et les Confrères qui vont vous voir, d'entendre les cris et le tapage des enfants. Et aussi quelle perte de temps pour vous : comment pouvez-vous faire librement vos exercices de piété, et travailler avec aise au règlement de vos affaires de fabrique et de paroisse ${ }^{10}$ ?"

Les évêques, du reste, redoutent toujours que la présence de parents laïcs ne détourne de leurs responsabilités les curés. De temps à autre, surtout dans la seconde moitié du $19^{\mathrm{e}}$ siècle, à l'heure où l'Église multiplie ses institutions et l'éventail de ses champs d'intervention, ils rappellent aux prêtres que l'argent tiré de leurs charges doit être "bien employé ": il ne devrait pas servir à faire vivre la famille, mais devrait plutôt financer les œuvres de l'Église. "Cessez donc de dépenser en faveurs inopportunes des revenus que vous pouvez employer à des fins pressantes de religion ", se fait dire, dans les années 1860 , un curé qui héberge neveux et nièces ${ }^{11}$. Les legs testamentaires, quant à eux, doivent avant tout favoriser les intérêts de l'Église ${ }^{12}$.

Les interventions épiscopales pour régler le sort des parents du curé logés au presbytère donnent cependant des résultats mitigés. Plusieurs prêtres, en effet, ne peuvent se résoudre à laisser partir une vieille mère, un frère infirme ou une sœur célibataire et sans soutien, fut-il d'une personnalité peu amène ou d'une conduite parfois douteuse. L'affection filiale

8. Archives de l'évêché de Saint-Hyacinthe (AESH), RL, 4, 211-212, J. La Rocque à F.X. Brunet, 12 septembre 1863.

9. L'image paisible et bucolique du presbytère est rapidement évoquée dans « Doux souvenir de mon village ", chanson publiée dans Nouvelle lyre canadienne. Recueil de chansons canadiennes et françaises, Montréal, Beauchemin, 1885, 190 : « Tout près de l'humble presbytère, asile d'un bon vieux curé..."

10. AESH, RL, 8, 250, Moreau à Pelletier, 28 septembre 1877.

11. AESH, RL, 4, 220, J. La Rocque à O. Monet, Saint-Grégoire, 23 septembre 1863.

12. En 1840, Bourget presse ainsi un prédicateur de retraite d'inciter les prêtres à faire de " bons testaments " pour que le " patrimoine du crucifix " ne tombe pas " entre les mains des laïcs ". Archives de la chancellerie de l'Archevêché de Montréal (ACAM), 901.042. M ${ }^{\text {gr }}$ Bourget, Retraites du clergé. 
et familiale se conjugue mal avec les intentions des évêques désireux d'instaurer l'idéal d'un corps clérical hiérarchisé, mais uni et solidaire, entretenant des rapports distants, quoique bienveillants, avec les laïcs. En 1836, le curé Demers répond, avec une candeur certaine, à l'évêque Lartigue qui le presse d'intervenir auprès de ses sœurs réputées insupportables : " Je suis encore à savoir ce que mes sœurs ont fait pour rendre ma maison inaccessible aux voisins et pas tenable pour les vicaires. Si vous voulez que je mette ordre à ce qui leur est reproché, ayant la bonté de m'articuler les faits qui les mettent dans leur tort ${ }^{13}$."

\section{Une coexistence difficile}

Les démêlés de l'abbé Demers, éprouvés, il faut le dire, par plusieurs autres prêtres à la même époque, indiquent bien les difficultés que pose la cohabitation d'hommes et de femmes de conditions et de personnalités différentes. L'attribution des postes de curés et de vicaires est soumise à des impératifs nombreux. Le personnel pastoral disponible est parfois restreint. Dans ces circonstances, l'évêque peut difficilement faire des tempéraments des personnes mises en présence le critère premier de ses choix. Comment pourrait-il, au demeurant, prévoir l'évolution des relations entre un curé, son vicaire et le reste de la maisonnée? La patience des uns et des autres est parfois mise à rude épreuve dans cette coexistence forcée. Certains curés trouvent plus sage de renoncer aux services des vicaires. L'un d'eux considère que lui et ses parents devraient modifier leurs habitudes de vie et " tenir une meilleure table " s'il devait prendre un assistant. Rechignant à faire cette dépense, le curé préfère continuer à assumer seul le ministère pastoral ${ }^{14}$. Ailleurs, il faut trouver moyen de vivre ensemble, malgré les différends. Pour mettre un terme à la situation conflictuelle qui envenime la vie au presbytère, un curé croit bon de mettre par écrit un certain nombre de remarques et de précisions dans un document intitulé « Remarques à son vicaire ». Le texte s'ouvre sur des considérations générales :

"Je tiens maison, tant bien que mal, depuis 35 ans. J'ai contracté certaines habitudes de vie dans ma maison et je ne puis pas les abandonner entièrement pour adopter des habitudes du vicaire quelques louables qu'elles soient ni obliger le personnel de ma maison à changer au gré du désir du vicaire ${ }^{15}$."

Le reste du document est à l'avenant. Il explique que le vicaire doit se plier aux coutumes de la maison : il doit "marcher dans les traces du curé".. Celui-ci refuse de déroger à sa manière de faire les confessions et les visites aux malades et dénonce l'inconséquence de son collaborateur. " Agir par

13. AESH, XVII, c.25, Demers à Lartigue, 19 septembre 1836.

14. AESH, XVII c.15 Waterloo, 22 avril 1868.

15. Archives de l'archevêché de Sherbrooke, FP-14, Coaticook, Gosselin à Chalifoux, circa 1893. 
caprice, écrit-il, c'est indigne d'un prêtre [...] On le pardonne dans un enfant ou dans une femme mais jamais à un homme et à plus forte raison jamais dans un prêtre. " Le texte contient aussi un passage relatif aux domestiques :

«Les serviteurs sentent assez leurs conditions pénibles sans qu'il y ait aucun besoin de notre part de leur faire sentir d'avantage ni faire notre possible pour la leur rendre plus pénible et plus odieuse et ces pauvres gens ont quelques fois autant de cœur que nous et tiennent à leur réputation et ils sont plus peinés quand elle est attaquée par un prêtre et surtout quand ils font tout leur possible pour plaire ${ }^{16}$."

Les sources n'expliquent pas davantage l'objet du litige entre le curé et le vicaire, ni le rôle qu'ont eu à jouer les employés de la maison dans cette dispute. On sait seulement que le vicaire conteste une des exigences de son supérieur le sommant de répondre aux visiteurs en son absence. Aux yeux du jeune prêtre, le curé est inflexible et a des attentes démesurées. Pendant tout le $19^{\mathrm{e}}$ siècle, d'autres vicaires posent des jugements semblables sur leur patron.

Le nom de certains curés réputés particulièrement exigeants ou colériques fait en effet frémir les jeunes prêtres. Les archives conservent plusieurs histoires de rapports difficiles qui expriment bien les tensions que suscite au quotidien l'organisation pyramidale de l'Église soumettant le vicaire au curé et les laïcs aux prêtres. Les sensibilités individuelles ne font pas toujours bon ménage avec les devoirs et les obligations qu'impose, dans les relations interpersonnelles, la vision d'un ordre hiérarchisé. L'abbé Demers évoqué plus haut juge sévèrement les différents vicaires qui se succèdent à ses côtés et qui ne semblent pas trouver grâce, non plus, aux yeux des sœurs du curé. Des vicaires traînent par ailleurs avec eux la réputation d'être légers, irresponsables ou instables, de se montrer susceptibles ou peu enclins à se plier à l'autorité. Les prêtres déchus, qui doivent à nouveau faire leurs preuves comme vicaires auprès d'un curé d'expérience, trouvent souvent en ce dernier du paternalisme ou de la défiance, quand ce n'est pas un sentiment d'antipathie à peine voilé. De telles préventions suffisent à ruiner le fragile lien de confiance et de respect nécessaire au partage de l'autel comme à celui de la table et du gîte. Des conflits entre les prêtres d'une même paroisse deviennent intenables. En 1874, un vicaire de la Gaspésie décrit les rapports qu'il entretient avec son supérieur immédiat :

"Ce qui me fait le plus de peine, c'est que M. Fournier [le curé] ne se gêne pas le moins du monde de m'abîmer d'injures devant les domestiques [...]. Il m'a chanté tout ce qu'il savait de bêtises, il m'a traité au dernier rang. Je vais vous en dire un peu, mais pas tout, car c'est impossible de me les rappeler : Vous êtes hypocrite (à plusieurs reprises), tête croche, sans génie, sans cœur, sans esprit, que je fais à ma tête, que je ne sais pas vivre [...] Il m'a dit hier "J'ai pas besoin de Monseigneur pour vous renvoyer ${ }^{17}$ ". "

16. Ibid.

17. Archives de l'évêché de Gaspé (AEG), III : 7,3, Mont-Louis, E. Côté à Langevin, 24 novembre 1874. 
Les relations entre les deux hommes sont si détériorées que l'évêque n'a d'autre choix que d'assigner ailleurs le jeune prêtre. Le curé d'une autre paroisse gaspésienne n'attend pas, quant à lui, que son supérieur réagisse et prend seul la décision de renvoyer son vicaire. La mésentente, devenue publique, mobilise les paroissiens qui prennent partie, les uns pour le curé, les autres pour son subalterne congédié ${ }^{18}$. Dans le contexte de vive excitation qui caractérise la paroisse, secouée par des querelles politiques, les démêlés des occupants du presbytère avivent les tensions. Le vicaire et, bientôt, le curé doivent partir. Des prêtres extérieurs à ces conflits sont envoyés sur place pour assurer le service du culte. Le presbytère et la paroisse pourront ainsi, espère-t-on, retrouver la quiétude d'antan.

\section{Madame curé}

La surveillance qui s'exerce sur les ménagères est, elle, particulièrement étroite. Au $18^{\mathrm{e}}$ siècle, pendant tout le $19^{\mathrm{e}}$ siècle, et même encore au siècle suivant, les évêques rappellent aux prêtres les règles qui doivent présider à l'engagement des servantes et à leurs relations avec les autres habitants du presbytère, en particulier les ecclésiastiques. Les servantes, écrit Pontbriand, l'évêque de Québec, en 1751, doivent avoir " l'âge prescrit par les canons ", c'est-à-dire 40 ans ${ }^{19}$. Pendant plus d'un siècle, la même exhortation est souvent reprise par ses successeurs et par les évêques des autres diocèses québécois qui portent une attention particulière à la question lors des visites pastorales. Aux réprimandes de leur supérieur, maints curés répondent qu'il est difficile de trouver une servante qualifiée ayant l'âge de 40 ans. Des dérogations à la règle sont donc autorisées. On tolère aussi que des proches parentes fassent office de ménagères. En 1851, le premier concile de Québec propose toutefois d'assujettir l'engagement de ces dernières à une règle interdisant leur présence dans les presbytères si elles ont entre douze et vingt-cinq ans. Les curés, estime-t-on, auraient alors trop à faire pour veiller sur elles et surveiller leurs agissements. Leurs fréquents voyages les empêcheraient en outre de s'acquitter convenablement de cette tâche. La présence de jeunes filles au presbytère est aussi jugée dangereuse, car " elle risquerait de faire parler les gens quand des confrères viendraient les visiter ${ }^{20}$ ". Ces avis, on l'a vu, rencontrent toutefois une oreille assez distraite chez plusieurs prêtres.

18. AEG, III : 7,3, Cap-Chat, T. Côté à Langevin, 14 décembre 1885.

19. Mandement de Mgr de Pontbriand pour le jubilé en date du 22 novembre 1751, cité dans le Recueil d'ordonnances synodales et épiscopales, 1859, p. 145. Voir aussi GaGnon, Serge, Plaisir d'amour et crainte de Dieu : sexualité et confession au Bas-Canada, SainteFoy [Québec], Presses de l'Université Laval, 1990, p. 22-23. Dans le diocèse de Rimouski, l'âge requis fut abaissé à 35 ans. "Circulaire au clergé, 5 septembre 1878 ", Mandements, lettres pastorales, circulaires de Mgr. Jean Langevin et statuts synodaux du diocèse de Saint Germain de Rimouski, de mai 1878 à mai 1887, disposés par ordre chronologique, Rimouski, Impr. A. G. Dion, 1889, 5, VI-21,9.

20. GrISÉ, Jacques, Les Conciles provinciaux de Québec et l'Église canadienne, 1851-1886, Montréal, Fides, 1979, p. 90-91. 
Le seuil de 40 ans, si cher aux évêques, traduit sans doute une conception fort répandue de la jeunesse associée à la beauté, à la sensualité et à la séduction. L'épiscopat est soucieux d'éviter à ses curés les tentations charnelles que pourrait imposer la cohabitation avec une nubile. " Il y a de graves inconvénients, note Bourget, évêque de Montréal, à ce que les jeunes filles demeurent dans les presbytères. C'est l'âge où elles se laissent facilement aller à la vanité, au plaisir, aux fréquentations ${ }^{21}$. " Enjôleuses qui corrompent les clercs, les jeunes filles sont également frivoles, volages ou trop naïves pour résister aux assiduités des galants. Au mieux, si elles ne font pas tomber les prêtres, elles suscitent les visites assidues de prétendants, dont la présence au presbytère est tout sauf souhaitable, la maison curiale ne devant jamais perdre le caractère de gravité que l'Église souhaite associer au clergé. Dans tous les cas, les langues s'excitent facilement à l'idée qu'une jeunesse réside au presbytère : " Encore une fois, répète Bourget, point de jeunes filles dans les presbytères pour faire des amours et exciter des cancans dans la Paroisse, aux dépens du curé ${ }^{22}$."

Mais sans doute faut-il voir dans la règle des 40 ans révolus encore plus qu'une simple manifestation des préjugés sur la jeunesse. On peut en effet penser que ce seuil répond aussi au désir de restreindre autant que possible les risques de grossesse encourus par d'éventuels manquements à la règle du célibat ecclésiastique. Au seuil de la ménopause, la fertilité des femmes décroît. Les prêtres qui, à l'encontre de leurs vœux et de la surveillance qui s'exerce sur eux, manqueraient à l'idéal de chasteté, éviteraient, à tout le moins, d'être compromis par une grossesse scandaleuse. Car sans être fréquente, la situation se produit parfois. En 1857, un prêtre doit quitter la paroisse du Dunham, dans les Cantons de l'Est, quand la grossesse de sa servante devient publique. Choqués de la situation, les paroissiens écrivent à l'évêque pour demander le retrait du curé qui poursuit sa carrière dans le lointain diocèse d'Hamilton ${ }^{23}$.

La ménagère, il est vrai, est un personnage qui suscite l'attention dans l'univers paroissial. Dans son recueil à saveur terroiriste, Vieilles Choses, Vieilles gens, Georges Bouchard la présente comme l'une des figures marquantes de la société villageoise. Sa description, où percent l'ironie et les préjugés, en fait " un type redouté ou respecté dans nos campagnes ${ }^{24}$ ". La servante de curé, l'un des rares personnages féminins, avec la "maîtresse d'école " et la " cousine des États ", que dépeint Bouchard, est " sévère ". Elle est " plus rigide qu'un article de droit canonique ". Elle a " des regards inquisiteurs sans cesse jetés sur autrui " et un nez qui a "l'air d'un guillemet sur une bouche à l'impératif ». Directive, elle s'approprie le presbytère

21. ACAM, RLB, 6, 542, Bourget à Cusson, 30 septembre 1851.

22. Ibid.

23. AESH, RL, 2, 199, Prince à Fitzgerald, 24 février 1857 et RL, 2, 211, Prince à Leclaire, 6 avril 1857.

24. Bouchard, Georges, Vieilles Choses..., Vieilles Gens. Silhouettes campagnardes, Montréal, Beauchemin, 1926, p. 49-56. 
et son mobilier, usurpe l'autorité de son maître, le curé, sur lequel elle veille néanmoins jalousement. L'opiniâtreté dont l'affuble l'écrivain défie en quelque sorte l'ordre social et religieux qui soumet la domestique à son maître, la femme à l'homme, le laïc au clerc. Quelques lettres trouvées dans la correspondance diocésaine reproduisent les mêmes stéréotypes sur la ménagère. La servante, y lit-on, est autoritaire, indiscrète, commère ou carrément malveillante. Des lettres de dénonciation l'accusent aussi d'entretenir des rapports trop étroits, voire libidineux, avec le curé. Du reste, les plaisanteries qui en font une "Madame Curé " témoignent de l'hégémonie du couple comme modèle et de la difficulté de penser le célibat, féminin surtout, à cette époque. Pour une large part, les descriptions de ménagères renvoient aux préjugés sur les "vieilles filles " qui fleurissent dans le discours littéraire du $19^{\mathrm{e}}$ siècle ${ }^{25}$. Ces plaisanteries et les accusations plus sérieuses qui sont parfois lancées ébranlent toujours la dignité du prêtre. Elles s'appuient quelquefois sur de solides soupçons, mais elles peuvent aussi, dans des contextes de crise, s'inscrire dans une stratégie de contestation de l'autorité du clergé. Au $19^{\mathrm{e}}$ siècle, la diffamation de la vertu féminine atteint l'homme qui est lié à cette femme, qu'il en soit l'époux ou, comme c'est le cas ici, le patron. Les doutes qui pèsent sur la vertu de la servante minent la réputation du prêtre.

\section{Un état inférieur}

Compte tenu de ces problèmes, des railleries, des commérages et du scandale, pourquoi l'Église recourt-elle à cette main-d'œuvre féminine? D'abord parce que l'emploi de domestiques apparaît nécessaire au clergé. Pasteur des âmes, médiateur entre la divinité et les hommes, le prêtre ne saurait s'abaisser à entretenir lui-même sa maison. Il emploie de préférence des femmes puisque les tâches ménagères sont traditionnellement du domaine féminin. Mais il y a plus. L'emploi de femmes dans les presbytères découle de motifs financiers. C'est du moins ce que laissent entendre les délibérations du $1^{\mathrm{er}}$ concile provincial de Québec, en août 1851, tandis qu'on s'interroge sur les problèmes causés par la présence de femmes auprès des curés et vicaires et sur la nécessité d'avoir des servantes. La question soumise aux commissaires est la suivante : "Doit-on défendre sous peine de suspense au clergé de cette Province de loger chez eux des servantes à moins qu'elles n'aient l'âge de 40 ans et soient au-dessus de tout soupçon, même s'il s'agit de leur mère, sœur ou nièce? "L'interrogation en sous-tend une autre : ne serait-il pas préférable d'engager des serviteurs pour entretenir le presbytère? Les commissaires répondent à cette dernière question par la négative. L'emploi de femmes est indispensable en raison des qualités d'ordre et de propreté qui les distinguent. Le recours à une main-d'œuvre

25. DAUPHIN, Cécile, "La vieille fille, histoire d'un stéréotype ", dans FARGE, Arlette, et KLAPISH-ZuBER, Christiane (dir.), Madame ou Mademoiselle? Itinéraires de la solitude féminine, $18^{e}-20^{e}$ siècles, Paris, Arthaud-Montalba, 1984, p. 207-231. 
féminine répond aussi à un souci d'économie. Les hommes, ajoutent en effet les commissaires, coûtent plus cher que les femmes.

On sait néanmoins peu de chose de la rétribution et des conditions de vie des servantes de curés. En général, les servantes de curés sont des célibataires, bien que certaines femmes mariées logeant au presbytère avec leur conjoint, " homme engagé du curé ", remplissent parfois cette fonction. La ménagère voit à l'entretien ménager du presbytère, prépare les repas et, en plusieurs endroits, cultive le potager et s'occupe de la bassecour du curé. Cette domesticité d'un type un peu particulier a laissé peu de traces dans les archives. Rares sont celles qui comme Adeline Malouin prennent la plume pour protester contre une décision jugée injuste et préjudiciable à leur avenir. Dans les années 1870, cette servante, fille de bonne famille âgée d'une trentaine d'années, travaille pour le curé de Cap-desRosiers, en Gaspésie. En 1874, des visiteurs de passage dans la paroisse signalent à l'évêque que la demoiselle scandalise les paroissiens par ses fréquentations ${ }^{26}$. Ces commérages poussent le supérieur diocésain à muter le curé et à l'obliger à renvoyer sa ménagère. En apprenant la nouvelle de son congédiement, Adéline adresse à $\mathrm{M}^{\mathrm{gr}}$ Langevin une première lettre qui exprime bien la vulnérabilité caractérisant l'existence des ménagères :

"Ces accusations sur lesquelles vous fondez la grande punition que vous m'imposez bien subitement me déshonorent complètement aux yeux du public, aux yeux de ma propre famille et de mes amis. Par là vous ôtez à une pauvre orpheline tous droits de vivre dans l'estime du monde, et conséquemment le moyen de gagner ma vie honorablement comme par le passé [...] Si vous ne me permettez pas de continuer à demeurer pour conservation de mon honneur avec Mr le curé, veuillez bien me trouver une situation semblable afin que je prouve par ma conduite que de tels bruits désagréables sont tout à fait faux ${ }^{27}$."

Cette lettre, restée sans réponse, est suivie quelques semaines plus tard d'une autre, marquée par le dépit et la colère. Sans détour, la servante s'adresse au supérieur diocésain pour qu'il rétablisse sa réputation et qu'elle retrouve sa dignité et un gagne-pain :

"Puisque vous n'avez pas eu l'obligeance de me dire pour quelle raison vous me chassez ignominieusement d'un presbytère, m'ôtant par là ma réputation, me faisant passer pour une fille de mauvaises mœurs, je vous avertis sérieusement que vous aurez à le constater devant les tribunaux civils $[\ldots]^{28}$."

Cette intervention et celle de son employeur, le curé Séguin, qui proteste que la demoiselle Malouin a " pour seul défaut d'être dûment canonique " et " polie $^{29}$ ", n'adoucissent guère l'évêque, qui refuse de revenir

26. Archives de l'archidiocèse de Rimouski, RLG, F, acte 477, Langelier à Séguin, 12 septembre 1874 .

27. AEG, III : 7,3, Cap-des-Rosiers, Adeline Malouin à Langevin, 23 septembre 1874

28. AEG, III : 7,3, Mont-Louis, A. Malouin à Langevin, 15 novembre 1874.

29. AEG, III : 7,3, Mont-Louis, Séguin à Langevin, 29 octobre 1874. 
sur sa décision. Adeline quitte donc son patron. Outré par l'attitude de son supérieur qu'il juge dur, injuste et inflexible, se disant incapable de trouver dans son village gaspésien une ménagère compétente, le curé menace à son tour de retourner à Montréal, son diocèse d'origine. Il tient pour " inacceptable " la vie solitaire que lui impose la décision de l'évêque. L'abbé Séguin obtient finalement d'être retiré de ses fonctions dans le diocèse de Rimouski et part pour les États-Unis ${ }^{30}$.

Pour exceptionnel qu'il soit, l'épisode est néanmoins révélateur des rapports qui se nouent dans la maison curiale. Il met en lumière la dépendance réciproque qui fonde les relations qu'entretiennent le curé et sa servante. Le statut du premier appelle en effet la présence dans les presbytères d'une main-d'œuvre plus ou moins abondante. Comme nous l'avons vu, la position particulière du prêtre au sein de la paroisse et ses responsabilités requièrent l'emploi de domestiques. Avec cette main-d'œuvre, les curés développent des rapports de confiance, et même parfois, à l'encontre des directives de l'Église, une complicité étroite. Femmes célibataires au statut incertain dans la société du $19^{\mathrm{e}}$ siècle, les servantes ont quant à elles une existence marquée par la précarité. Leur survie économique est tributaire tout à la fois de ce que pensent d'elles leur patron, le curé, mais aussi ce qu'en disent l'opinion publique et l'évêque du diocèse. Ainsi, cette dépendance réciproque liant curé et ménagère n'implique pas des rapports égalitaires. En tout temps, la domestique doit se rappeler son état et son statut inférieurs face au curé, chef suprême du presbytère comme de la paroisse. "Que Madame ou Mademoiselle la gouvernante gouverne sa cuisine, c'est très bien, mais toujours sous le règne du curé; mais qu'elle ne gouverne jamais le curé en quoi que ce soit. Accoutumons la à ne jamais se mêler des affaires de la paroisse et qu'elle reste bien dans son rôle ", note un curé, dans son journal personnel, à la fin d'une retraite sacerdotale, en août $1883^{31}$. Vers la même époque, un autre prêtre écrit dans ses mémoires personnels qu'il " est indécent qu'un curé se laisse dominer pas sa ménagère ". D'excellents curés, précise-t-il, " ont perdu de l'estime de leurs confrères, ainsi que de certains paroissiens quand Julie, répondait, par exemple : on ne chante pas de messes cette semaine ${ }^{32}$ ". La servante idéale ne se mêle donc pas des affaires de la paroisse. Elle est ordonnée, propre, discrète, modeste, réservée et soumise. Elle évite toute sensualité, tout comportement pouvant être interprété de manière équivoque ${ }^{33}$.

30. Morin, A.-Cléophas, Dans la Maison du Père. Nécrologie sacerdotale du diocèse de Rimouski 1867-1967, Rimouski, [s.n.], 1967, p. 17.

31. Roy, Jean et Hudon, Christine (prés.), Le journal de Majorique Marchand, curé de Drummondville, 1865-1889, Sillery [Québec], Septentrion, 1994, p. 157

32. Archives du Séminaire de Saint-Hyacinthe, AFG 41 : Pierre-Athanase Saint-Pierre, 1.76, Saint-Athanase.

33. Une description de la servante idéale figure dans le roman d'Eugène RivEsT, Roman d'un curé de campagne, Montréal, Éditions de la Vallée, 1963. L'œuvre, bien que postérieure à l'époque étudiée ici, fait écho au discours de l'Église sur les rôles sociaux et sur la place du clergé déjà présent au $19^{\mathrm{e}}$ siècle. Son auteur, lui-même prêtre, insiste sur les qualités exemplaires de la ménagère Prudence Noiseux. 
Cette définition des rôles et des modèles de comportement qui doivent voir le jour au presbytère est implicitement remise en question en 1904 par le roman Marie Calumet du journaliste Rodolphe Girard. Ce roman, inspiré d'une chanson traditionnelle, raconte, en une vingtaine de chapitres, le quotidien du presbytère de Saint-Ildéphonse. La ménagère, Marie Calumet, une femme de 40 ans, est énergique, organisée et entreprenante. Elle a un sens pratique très poussé et une bonne dose de jugement. Bientôt, le curé Flavel, un homme simple et bon, mais naïf et un peu empoté, s'en remet à elle pour la gestion de ses affaires et la consulte pour toutes les décisions qu'il doit prendre. Marie devient ainsi indispensable à son patron et commence à exercer un grand ascendant dans la paroisse où elle fait l'objet d'une profonde estime.

Le livre remporte un certain succès auprès du public, qui en achète plusieurs exemplaires. La presse, religieuse notamment, lui réserve quant à elle un accueil fort mitigé auquel fait écho une lettre circulaire au clergé signée par $M^{\text {gr }}$ Bruchési, l'archevêque de Montréal, qui juge l'ouvrage « aussi grossier qu'immoral et impie " et signale que " les lois de l'Index en interdisent la lecture ${ }^{34}$ ". Comme l'ont souligné bon nombre d'études, plusieurs éléments du livre, entre autres son ton irrévérencieux, ses accents scatologiques, la sensualité qui se dégage de Marie Calumet, femme ronde, bonne vivante, affublée de vêtements aux coloris éclatants, étaient de nature à attirer les foudres de l'archevêque. Mais on peut penser que la réaction de Bruchési découle aussi de l'image même du clergé que propose le livre. Dans le roman, le prêtre, loin d'être un homme exceptionnel, éminemment digne et différent de ses paroissiens, se présente comme un être assez ordinaire, qui s'efface derrière la figure charismatique d'une femme, sa servante. Ce renversement des rôles, qui désacralise le personnage du curé, figure sans doute au nombre des facteurs ayant conduit à la condamnation du roman. L'influence d'une institution comme l'Église dépend notamment de l'image qu'elle projette, d'où l'importance de cultiver, de rectifier ou d'interdire tout ce qui met en péril sa réputation et son ascendant.

Que révèle, en définitive, ce tour d'horizon trop rapide? Revenons sur les intentions de départ de cet article. Il s'agissait d'étudier le presbytère comme un microcosme de l'univers paroissial, d'y déceler les représentations, ainsi que les préjugés qui marquent les différents états de vie et de mettre en lumière les tensions que ces situations inégales engendrent. Comme nous l'avons vu, les rôles et la place qu'occupent les membres de la maisonnée curiale sont strictement réglés. Ces maisons font l'objet d'une double régulation, celle d'un pouvoir extérieur à l'espace paroissial,

34. Cité par HÉBERT, Pierre et NicOL, Patrick, Censure et littérature au Québec. Le livre crucifié (1625-1919), Montréal, Fides, 1997, p. 133-134. Sur le roman, lire aussi VANASSE, André, "Marie Calumet ", dans LemiRe, Maurice (dir.), Dictionnaire des ouvres littéraires du Québec, t. 2, 1900-1939, Montréal, Fides, 1980, p. 673-676; WAYMEL, Marie-Claude, Dissidence idéologique et dissonance romanesque : le roman québécois de 1900 à 1920, Mémoire de maîtrise (études littéraires), Université du Québec à Montréal, 1981, 135 p. 
l'évêque, pouvoir officiel, et celle d'un pouvoir de l'intérieur, officieux celuilà, mais néanmoins efficace, celui des populations.

La vie au presbytère met par ailleurs à jour plusieurs tensions qui caractérisent l'Église et la paroisse québécoises du $19^{\mathrm{e}}$ siècle. Elle révèle tout d'abord des tensions entre le public et le privé, entre un esprit communautaire, qui cherche à imposer sa logique et sa morale, et la poussée de l'intimité, qui vise à circonscrire un espace privé. Au presbytère s'exprime aussi la difficulté de concilier, d'une part, l'idéal sacerdotal, qui commande des rapports d'autorité au sein du clergé, et d'autre part, les sensibilités individuelles. S'y manifeste aussi une tension entre ce même idéal sacerdotal imposant une ségrégation, ou du moins, une distance à préserver entre clercs et laïcs et l'attachement des prêtres pour leur famille. Les murmures et les commérages autour du quotidien des habitants de la maison curiale traduisent également un écart entre la morale sexuelle catholique axée sur la continence et la culture populaire, qui sans rejeter les enseignements de l'Église, ne réprouve ni les paillardises ni les grivoiseries viriles, expressions parmi d'autres d'un anticléricalisme latent et diffus. Enfin, ces mêmes cancans témoignent d'une tension, dans la société du $19^{\mathrm{e}}$ siècle, entre la règle du célibat ecclésiastique et le modèle hégémonique du couple, un modèle qui conduit les populations à considérer le curé et sa servante comme des conjoints. Ceci dit, il faut remarquer que le discours sur la chasteté ecclésiastique comme, du reste, celui sur le mariage sont tous deux porteurs de préjugés à l'égard des femmes, tantôt sensuelles et corruptrices de prêtres, tantôt vieilles filles revêches, indiscrètes, médisantes et autoritaires.

Dans la gestion de ces tensions et des problèmes auxquels elles donnent lieu, les interventions des évêques sont guidées par le souci de préserver les réputations. La dignité du clergé, l'honneur de l'Église poussent l'autorité diocésaine à émettre des avertissements et, au besoin, à sévir contre les curés dont les relations avec une servante ou avec des parents font problème. Car le scandale nuit non seulement à l'ecclésiastique incriminé, mais il entache plus largement la réputation de tout le clergé. À l'encontre du discours ecclésial, qui exalte le personnage du prêtre, le scandale en fait un homme ordinaire, soumis aux mêmes penchants, aux mêmes problèmes, aux mêmes aléas quotidiens que le commun des mortels. 


\section{RÉSUMÉ}

L'article s'intéresse aux relations qu'entretiennent entre eux les occupants du presbytère. Il montre que trois types de rapports inégalitaires peuvent y être observés : ceux, qui prennent place, d'abord, parmi le clergé, entre curé et vicaire, ceux perceptibles, à un second niveau, dans l'Église, entre clercs et laïcs, et enfin, ceux qui, au sein de la société du $19^{\mathrm{e}}$ siècle, unissent les hommes et les femmes. Il révèle également que les rôles et la place qu'occupe chacun sont strictement réglés et que la vie au presbytère met à jour plusieurs tensions qui caractérisent l'Église et la paroisse québécoises au $19^{\mathrm{e}}$ siècle.

\section{ABSTRACT}

This paper focuses on the relationships between the people living in nineteenth century Quebec Catholic presbyteries. One can observe three types of unequal relations in these: those between the parish priest and the curate, those between the clergymen and the secular members of the congregation and, finally, those between the men and the women. The relations of power between these various roles were strictly regulated. This paper demonstrates how presbytery life reflected the tensions that existed between nineteenth century Québécois and their religion. 